\title{
In Situ Raman Spectroscopy of COOH-Functionalized SWCNTs Trapped with Optoelectronic Tweezers
}

\author{
Peter J. Pauzauskie, ${ }^{1,2}$ Arash Jamshidi, ${ }^{3}$ Joseph M. Zaug, ${ }^{1}$ Sarah Baker, ${ }^{1}$ T. Y.-J. Han, ${ }^{1}$ \\ Joe H. Satcher Jr., ${ }^{1}$ and Ming $\mathrm{C}$. $\mathrm{Wu}^{3}$ \\ ${ }^{1}$ Chemical Sciences Division, Lawrence Livermore National Laboratory, 7000 East Avenue L-231, Livermore, \\ CA 94551, USA \\ ${ }^{2}$ Department of Materials Science and Engineering, University of Washington, Seattle, WA 98195, USA \\ ${ }^{3}$ Department of Electrical Engineering, University of California, Berkeley, CA 94720, USA \\ Correspondence should be addressed to Peter J. Pauzauskie, peterpz@uw.edu
}

Received 2 May 2011; Revised 2 November 2011; Accepted 16 November 2011

Academic Editor: Eric Pei Yu Chiou

Copyright (C 2012 Peter J. Pauzauskie et al. This is an open access article distributed under the Creative Commons Attribution License, which permits unrestricted use, distribution, and reproduction in any medium, provided the original work is properly cited.

Optoelectronic tweezers (OETs) were used to trap and deposit aqueous dispersions of carboxylic-acid-functionalized singlewalled carbon nanotube bundles. Dark-field video microscopy was used to visualize the dynamics of the bundles both with and without virtual electrodes, showing rapid accumulation of carbon nanotubes when optical virtual electrodes are actuated. Raman microscopy was used to probe SWCNT materials following deposition onto metallic fiducial markers as well as during trapping. The local carbon nanotube concentration was observed to increase rapidly during trapping by more than an order of magnitude in less than one second due to localized optical dielectrophoresis forces. This combination of enrichment and spectroscopy with a single laser spot suggests a broad range of applications in physical, chemical, and biological sciences.

\section{Introduction}

One persistent challenge in molecular sensing is the enriching of candidate analytes to concentrations high enough for detection. Optoelectronic tweezers (OET) recently have been used as a versatile platform for trapping objects such as polystyrene spheres, living cells [1], and solid-state nanowires [2], and both [3] single- and [4] multi-walled samples of carbon nanotubes using 100,000x less optical power than single-beam laser traps. Based on a combination of dielectrophoresis and optical image patterning, OET has the unique capability of massively parallel localization of organic and inorganic nanoscale structures for both direct visualization and spectroscopic characterization. In this paper, we use carboxylic-acid- (COOH-) functionalized single-walled carbon nanotubes as a model system to demonstrate analyte enrichment by over an order of magnitude with a low-power OET trapping laser that serves simultaneously as a Raman spectroscopic probe.

\section{Materials and Methods}

2.1. Carbon Nanotube Sample Preparation. COOH-functionalized carbon nanotubes have been used as a surfactantfree alternative for aqueous SWCNT suspensions, with the hydrophilic $\mathrm{COOH}$-surface functional groups serving as a means to suspend the nanotubes on polar solvents such as water [5]. In this work, $\mathrm{COOH}$ functionalized SWCNTs (P3, Carbon Solutions, Inc., $\sim 4$ atomic \% COOHfunctionalization) were dispersed as made in Milli-Q deionized water, bath-sonicated for $30 \mathrm{~min}$, and centrifuged for $30 \mathrm{~min}$ at $16,000 \mathrm{~g}$ to remove large bundles and other metallic catalyst particles yielding a semitransparent solution.

2.2. Carbon Nanotube Sample Characterization. Transmission electron microscopy (JEOL CM-300) and atomic force microscopy (Veeco) were used to characterize the physical dimensions for the nanotube bundles. Atomic force microscopy measurements provide a diameter distribution 


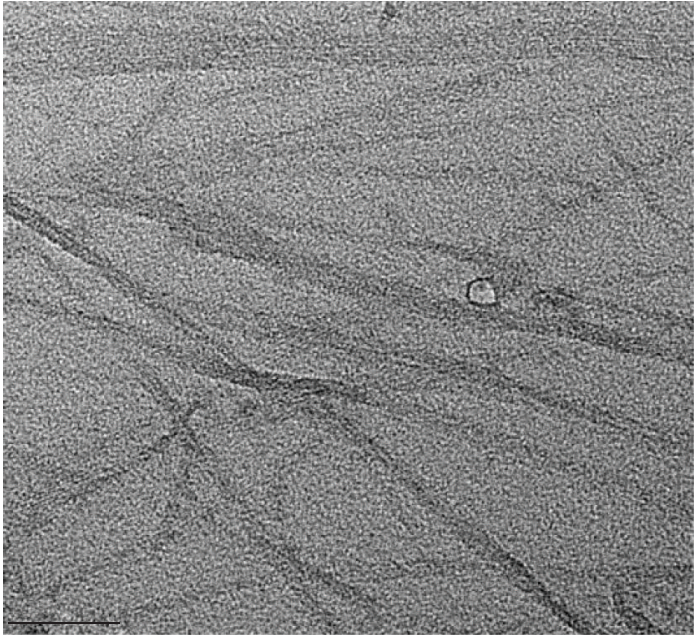

(a)

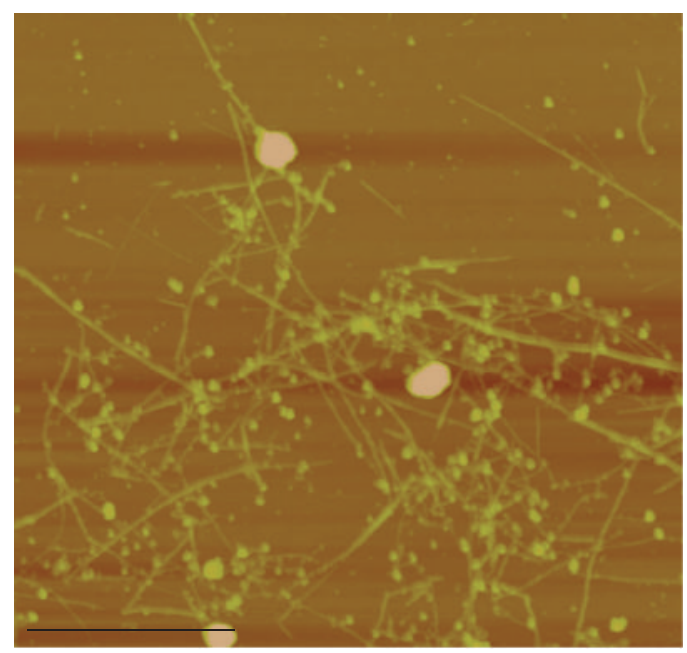

(b)

Figure 1: Characterization of COOH-functionalized SWCNTs used in this study. (a) Bright-field transmission electron micrograph. Scale bar $=50 \mathrm{~nm}$. (b) Atomic force micrograph of SWCNTs on an aminopropylesilane-coated mica wafer. Scale bar $=1 \mu \mathrm{m}$.

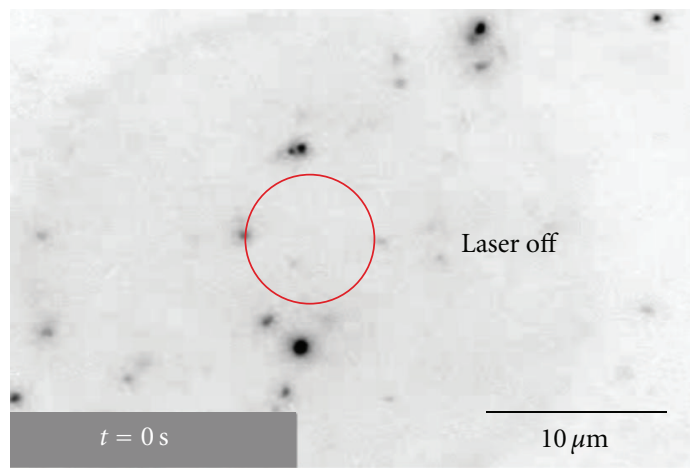

(a)

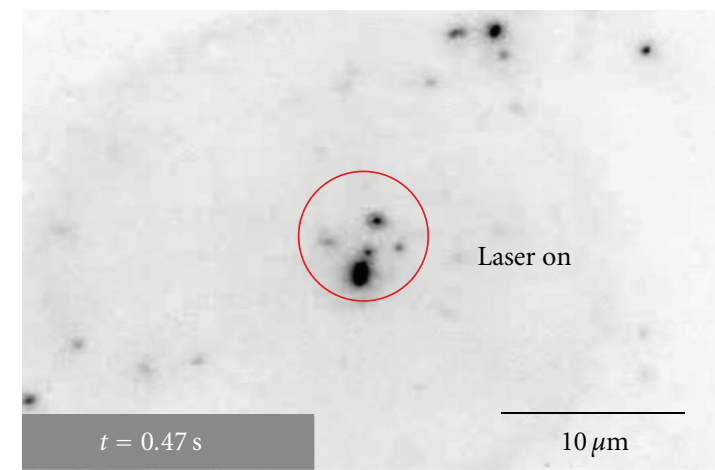

(b)

Figure 2: Dark-field EMCCD video microscopy of optoelectronic trapping of COOH-SWCNTs with laser off (a) and on (b) at an AC bias of $15 \mathrm{Vpp}, 100 \mathrm{kHz}$. Images are inverted to enhance visibility of faint light scattering from SWCNT bundles. The laser line (Ar $\left.{ }^{+}, 488 \mathrm{~nm}\right)$ is blocked by a holographic notch filter.

for the carbon nanotube bundles ranging from 2 to $11 \mathrm{~nm}$, and lengths ranging from 0.1 to $2 \mu \mathrm{m}$ (Figures 1 (a) and $1(\mathrm{~b})$ ), with a mean bundle diameter of $6.4 \pm 2.4 \mathrm{~nm}$.

\subsection{Dark-Field Imaging with Optoelectronic Tweezers. OET} chambers were fabricated as reported previously [1], and images were collected during trapping with a Nikon Eclipse LV150 microscope equipped with long working distance dark-field objective lenses. Dark-field optical microscopy was used [6] to image aqueous suspensions of SWCNT bundles with a video-rate, thermoelectrically cooled, backilluminated electron multiplying charge-coupled device camera (Andor, iXon). Standard chamber depths were fixed with double-sided adhesive tape with a standard thickness measured at 75 microns with a hand-held micrometer. During darkfield imaging, the trapping was performed with an argon-ion laser using the $488 \mathrm{~nm}$ emission line. The laser power was measured to be $100 \mu \mathrm{W}$ focused to a spot size of 5 microns. The laser spot is filtered from the dark-field image using a holographic notch filter.

2.4. Raman Microscopy. Raman measurements were made with the $632.8 \mathrm{~nm}$ line of a helium-neon laser. Laser light was focused onto samples using a long working distance $20 \mathrm{x}$ near-IR corrected apochromatic objective lens (Mitutoyo). Scattered light was collected using the same objective and was focused onto a pinhole (to improve spatial resolution) before being collimated and directed into the spectrometer. A holographic filter (Kaiser Optics) was used to reject elastically scattered laser light. The spectrometer was a $0.3 \mathrm{~m}$ Jobin Yvon LabRam system equipped with 1,200 lines/mm and 1,800 lines/mm gratings. The detector was an LN2cooled CCD (Roper Scientific). The system was calibrated 


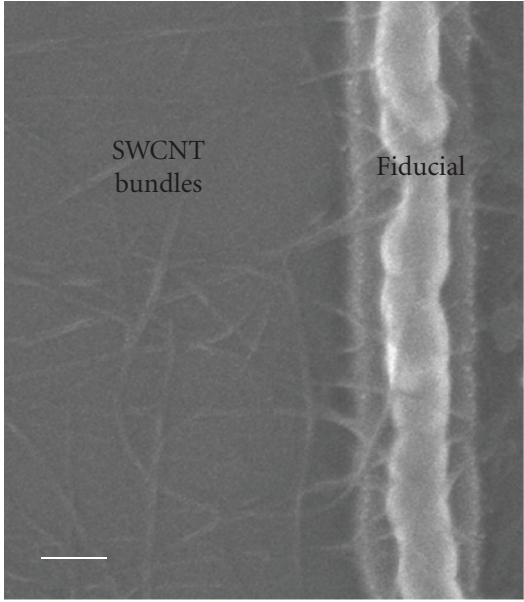

(a)

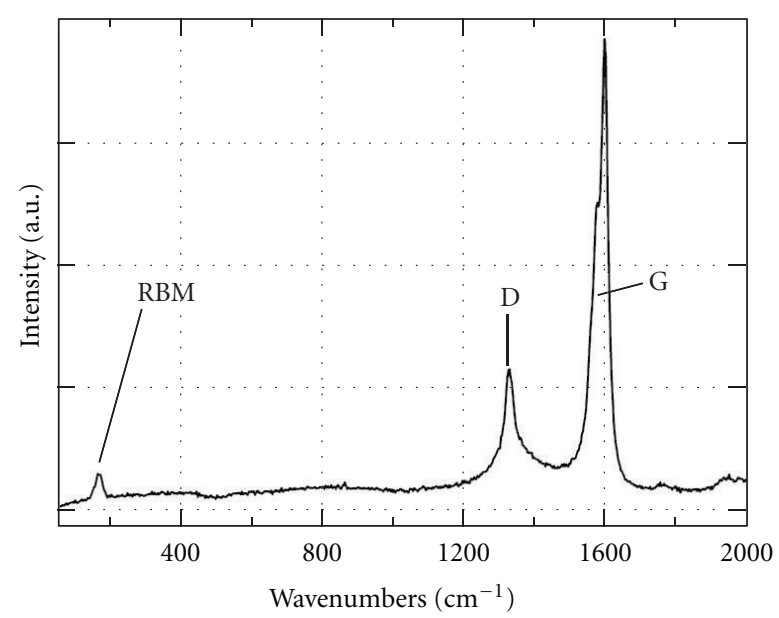

(b)

FIGURE 3: Ex situ characterization of COOH-SWCNTs following deposition with optoelectronic tweezers at high peak-to-peak voltages. (a) SEM image of SWCNT bundles deposited next to a Ti/Au fiducial marker. Scale bar $=100 \mathrm{~nm}$. (b) Raman spectrum of deposited SWCNT bundles showing radial breathing mode (RBM), D, and G bands; a-Si background subtracted.

using a neon lamp. In all experiments, laser power was measured with a hand-held power meter.

\section{Results and Discussion}

When a $15 \mathrm{Vpp}, 100 \mathrm{kHz}$ trapping voltage is applied across the OET chamber, the SWCNTs are observed to move away from one another (Figure 2(a)) due to repulsive, in-phase dipole-dipole interactions. When the trapping laser is turned on, SWCNT bundles are observed to migrate into the laser spot (Figure 2(b)), agreeing with previous reports of positive dielectrophoresis forces for metallic SWCNTs [7] as well as with recent calculations for MWCNTs predicting forces in the range of piconewtons [4]. At high trapping voltages $(20 \mathrm{Vpp}, 100 \mathrm{kHz})$, the SWCNTs are observed to attach irreversibly to the a-Si surface. Electron beam lithography is used to pattern a grid of metallic fiducial markers $(20 \mathrm{~nm} \mathrm{Ti}$, $50 \mathrm{~nm} \mathrm{Au}$ ) on top of the polished a-Si layer, which are then used to locate regions on the OET surface following Raman deposition experiments. Using scanning electron microscopy (SEM) (Figure 3(a)) and micro-Raman (Figure 3(b)) for ex situ characterization confirms that the deposited structures are indeed SWCNT bundles.

Single-beam laser tweezers have been used previously for three-dimensional trapping [8,9], and Raman characterization [10] of SWCNT suspensions; however, they typically require several milliwatts of power to produce electric field gradients sufficient for trapping. Confocal micro-Raman measurements may also be performed with OET in an upright backscattering configuration. Generally, several hundred $\mu \mathrm{W}$ from the polarized $632.8 \mathrm{~nm}$ line of a CW He: Ne laser (Figure 4(a)) are focused to an area of $\sim 75 \mu \mathrm{m}^{2}$, yielding a maximum local irradiance of $<500 \mathrm{~W} / \mathrm{cm}^{2}$, which is more than sufficient to actuate the OET virtual trapping electrodes while simultaneously providing enough inelastically scattered photons for Raman spectroscopy. The focal point is positioned in the chamber with a motorized translation stage, using the Raman signal of the a-Si as a means of ensuring that the Raman focal volume coincides directly with the OET-trapping volume.

When Raman spectra are taken from the $\mathrm{COOH}-$ SWCNT dispersions, signal levels are low due to both the low concentration of tubes in solution and defects within the carbon lattice that decrease the inelastic scattering crosssection [11]. Raman spectra are acquired both with and without the OET-trapping voltage $(6 \mathrm{Vpp}, 100 \mathrm{kHz})$ at both the a-Si/fluid interface (Figure $4(\mathrm{a}))$ and $\sim 20 \mu \mathrm{m}$ above the $\mathrm{a}-\mathrm{Si}$. Once the trapping voltage is applied, the in-plane $\mathrm{E}_{2 \mathrm{~g}}$ stretching mode at $1591 \mathrm{~cm}^{-1}$ collected from the a-Si/fluid interface shows an 18 -fold increase based on a ratio of integrated $1591 \mathrm{~cm}^{-1}$ peak areas. The enhancement exists both when compared to the case with the trapping voltage off, and with the voltage on, but with the focus $\sim 20 \mu \mathrm{m}$ above the a-Si surface (Figure 4(b)). The signal enhancement is reversible, depending only on whether the laser is present to generate inhomogeneous lateral electric trapping fields. When the AC field is turned off, the signal relaxes to the background level, suggesting that trapped SWCNTs become free to diffuse through solution in agreement with dark-field images (Figure 2).

\section{Conclusion}

In conclusion, we report here the first demonstration of simultaneous trapping and micro-Raman spectroscopy of SWCNT materials with OET, providing a versatile means for locally concentrating dilute samples, therefore, enhancing the detected Raman signals by over an order of magnitude. This signal enrichment can potentially be combined with other Raman enhancement techniques such as surfaceenhanced Raman spectroscopy (SERS) to create more sensitive Raman probes. OET is unique in that a few hundred 


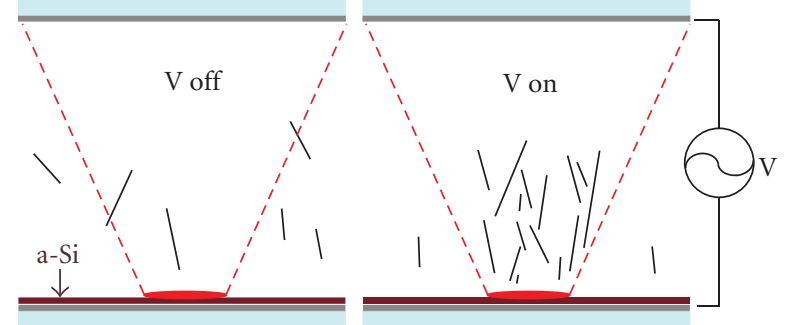

(a)

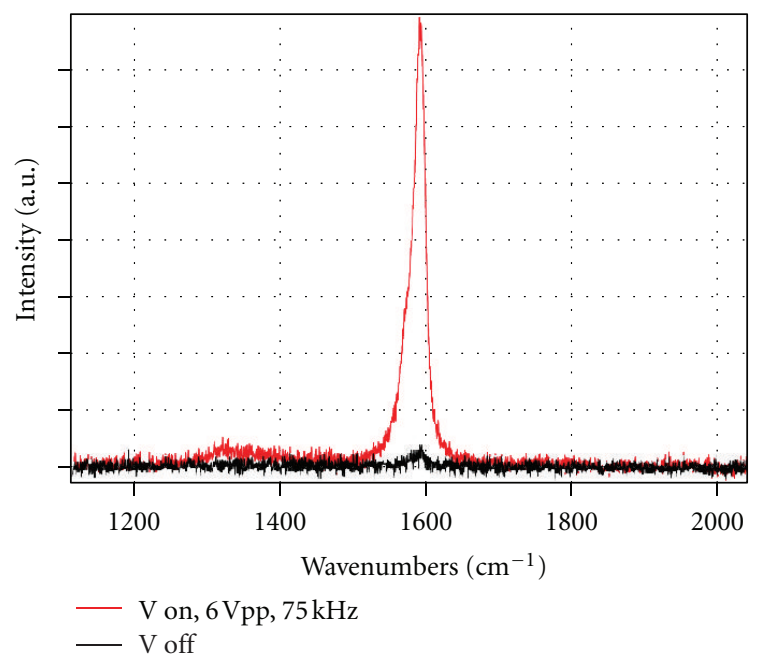

(b)

FIgURE 4: Enhanced Raman signal from COOH-SWCNTs trapped with OET. (a) Schematic of micro-Raman experiment and (b) Raman spectrum $\left(480 \mathrm{~W} / \mathrm{cm}^{2}, 60 \mathrm{~s}\right)$ with (red) and without (black) OET-trapping bias of $6 \mathrm{Vpp}, 75 \mathrm{kHz}$.

micro-Watt laser intensities can be used to enrich the local analyte concentration while simultaneously producing inelastically scattered photons for sample characterization. This combination of enrichment and spectroscopy with a single laser spot suggests a broad range of applications in physical, chemical, and biological sciences. Finally, these results will help guide future experiments with isolated single-walled carbon nanotubes.

\section{Acknowledgments}

P. J. Pauzauskie thanks the LLNL for a Lawrence Fellowship. This work was supported in part by the NIH Roadmap for Medical Research (Grant no. PN2 EY018228) and DARPA. The authors thank Hsan-Yin Hsu, Justin K. Valley, Steven L. Neale, and Marcus Worsley for valuable assistance. This work was performed under the auspices of the U.S. Department of Energy by Lawrence Livermore National Laboratory under Contract no. DE-AC52-07NA27344.

\section{References}

[1] P. Y. Chiou, A. T. Ohta, and M. C. Wu, "Massively parallel manipulation of single cells and microparticles using optical images," Nature, vol. 436, no. 7049, pp. 370-372, 2005.
[2] A. Jamshidi, P. J. Pauzauskie, P. J. Schuck et al., "Dynamic manipulation and separation of individual semiconducting and metallic nanowires," Nature Photonics, vol. 2, no. 2, pp. 86-89, 2008.

[3] M. W. Lee, Y. H. Lin, and G. B. Lee, "Manipulation and patterning of carbon nanotubes utilizing optically induced dielectrophoretic forces," Microfluidics and Nanofluidics, vol. 8, no. 5, pp. 609-617, 2010.

[4] P. J. Pauzauskie, A. Jamshidi, J. K. Valley, J. H. Satcher, and M. C. Wu, "Parallel trapping of multiwalled carbon nanotubes with optoelectronic tweezers," Applied Physics Letters, vol. 95, no. 11, Article ID 113104, 2009.

[5] K. Balasubramanian and M. Burghard, "Chemically functionalized carbon nanotubes," Small, vol. 1, no. 2, pp. 180-192, 2005.

[6] Z. Yu and L. Brus, "Rayleigh and Raman scattering from individual carbon nanotube bundles," The Journal of Physical Chemistry B, vol. 105, no. 6, pp. 1123-1134, 2001.

[7] R. Krupke, F. Hennrich, H. von Löhneysen, and M. M. Kappes, "Separation of metallic from semiconducting single-walled carbon nanotubes," Science, vol. 301, no. 5631, pp. 344-347, 2003.

[8] S. Tan, H. A. Lopez, C. W. Cai, and Y. Zhang, "Optical trapping of single-walled carbon nanotubes," Nano Letters, vol. 4, no. 8, pp. 1415-1419, 2004.

[9] O. M. Maragò, P. H. Jones, F. Bqnaccorso et al., "Femtonewton force sensing with optically trapped nanotubes," Nano Letters, vol. 8, no. 10, pp. 3211-3216, 2008.

[10] T. Rodgers, S. Shoji, Z. Sekkat, and S. Kawata, "Selective aggregation of single-walled carbon nanotubes using the large optical field gradient of a focused laser beam," Physical Review Letters, vol. 101, no. 12, Article ID 127402, 2008.

[11] R. Saito, M. S. Dresselhaus, and G. Dresselhaus, Physical Properties of Carbon Nanotubes, Imperial College Press, London, UK, 1998. 

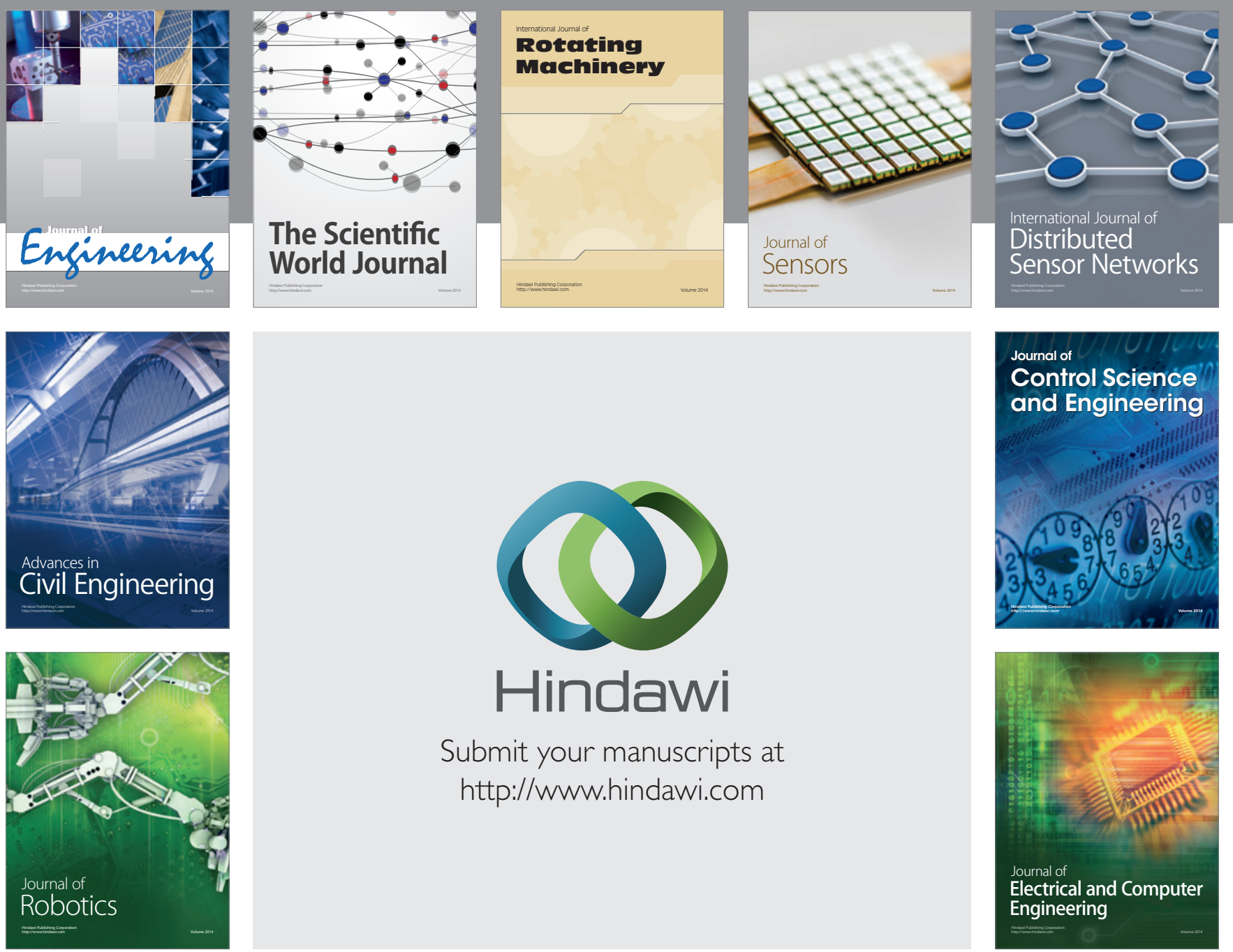

Submit your manuscripts at

http://www.hindawi.com
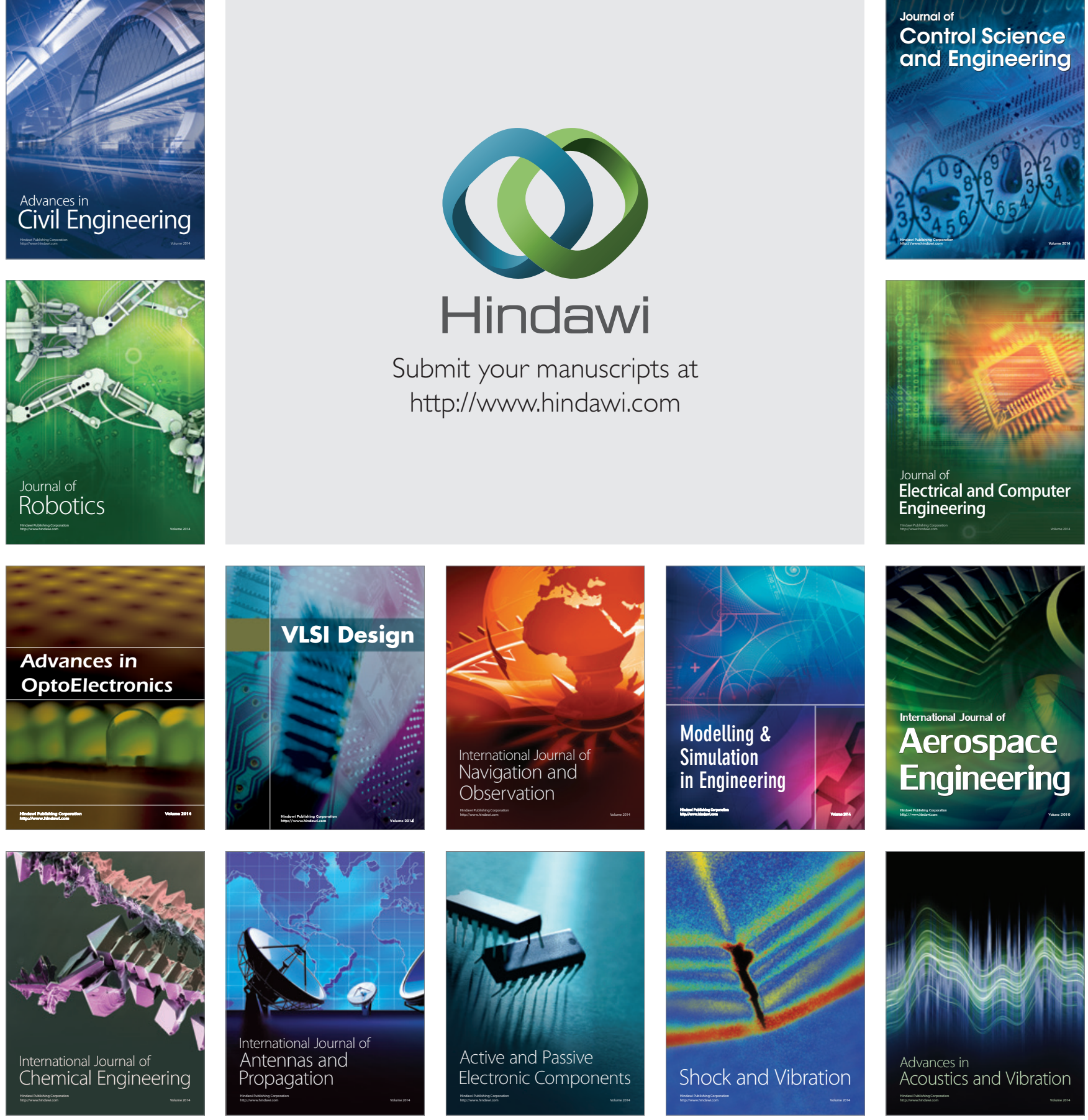